\title{
Combination of Osteogenesis imperfecta, Ehlers Danlos syndrome, TRAP syndrome, and Stickler syndrome as multiple inherited connective tissue disorders in a 28-year-old Egyptian woman.
}

\section{El-Saadany $H^{*}$ and Negm S}

Department of Internal Medicine and Rheumatology unit, Air Force Specialized Hospital, New Cairo, Egypt

\begin{abstract}
Collagen related diseases most commonly arise from genetic defects or nutritional deficiencies that affect the biosynthesis assembly posttranslational modification a secretion or other processes involved in normal collagen production. One thousand mutations have been identified in twelve out of more than twenty types of collagen. These mutations can lead to various diseases at the tissue level. This report describes an Egyptian female presented by multiple different polysystematic disorders proved to be manifestations of multiple rare inherited connective tissue disorders in the same patient to be the first woman worldwide to comprise all these rare syndromes up to our review.
\end{abstract}

Keywords: Collagen, Modification, Disorders, Tissues

Accepted on March 05, 2018

\section{Introduction}

Heritable disorders of connective tissues are a heterogeneous group of disorders characterized by abnormalities in skeletal tissues including cartilages abonesatendonsa Ligaments muscles and skin. These disorders originally described by Mckusicta have been classified on the basis of clinical findings and molecular criteria [1]. They are sub classified into disorders that primarily affect cartilage and bone (the skeletal dysolasias) and disorders that have a more profound effect on connective tissue including Ehlers Danlos syndrome and other disorders manifested abnormal extracellular matrix molecules [2]. We report a very rare case of multiple inherited connective tissue disorders in the same patient in a 28-year-old Egyptian woman with Ontogenesis imperfect a Ehlers Danlos syndrome a TRAP syndrome and Stickler syndrome.

\section{Case Report}

An Egyptian women was presented by severe recurrent vomiting abdominal paina melena and signs of mal absorption pain allover weakness of both lower limbs dizzy spills and vertigo [3]. She was made nothing per mouth (NPO) and received total parenteral nutrition (TPN).

The condition started at the age of 12 when she developed a right hip fracture after minor trauma a she was operated upon with a screw and plate. DEXA scan at that time revealed severe osteoporosis for which she is treated by calcitonin then bisphosphonates. Later she got a fracture patella assured fracture at the tarsal bones of the left foot with delayed healing. At the age of 20 she had a low back pain with fissure fracture at the lumbar vertebrae and disc prolapse [4].

At the age of 24, she developed severe microcytic hypochromic anemia amour endoscopy revealed multiple mucosal tears and gastric erosions. Later on she noticed black tarry stool with further drop in hemoglobin level and temporomandibular dislocation confirmed by CT scan. She developed a recurrent moderate degree fever with no response to the usual measures and also negative blood cultures and blood films for malaria the fever continued for four months without any proof of infection [5].

\section{Examination}

Short stature blue sclera waddling gaitaalooecia Universalist hypermobile joints stretchy skin enlarged skull depressed nasal bridge (Figures 1 and 2).

\section{Investigations}

* Serum calcium, phosphorus was within normal.

* PTH was mildly elevated.

* Moderate vitamin D deficiency.

* MRI spine showed cod fish appearance of the vertebrae (Figure 3).

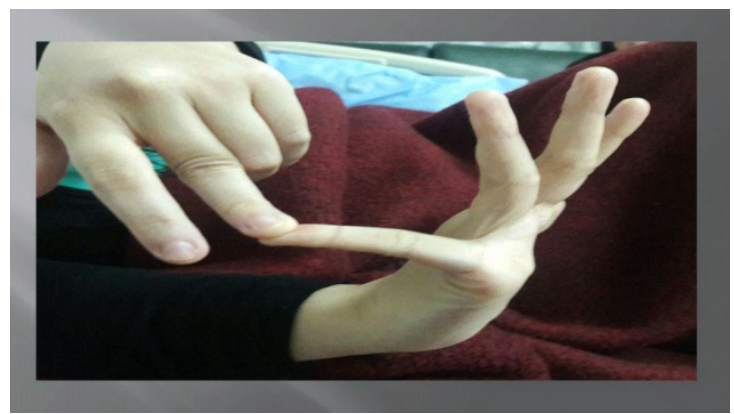

Figure 1. Hyoermobile joints.

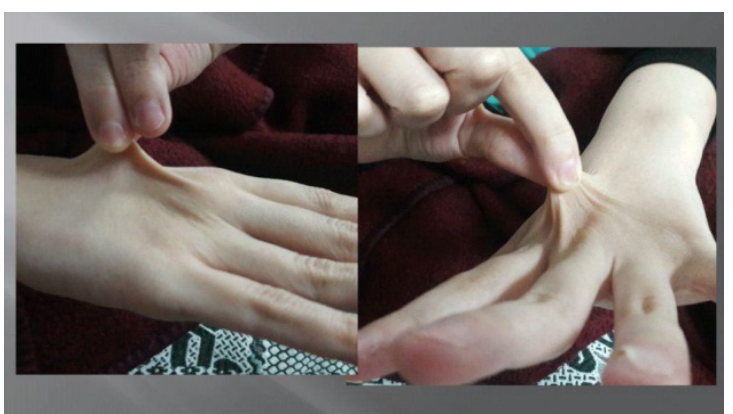

Figure 2. Elastic skin. 
Citation: El-Saadany H, Negm S. Combination of Osteogenesis imperfecta, Ehlers Danlos syndrome, TRAP syndrome, and Stickler syndrome as multiple inherited connective tissue disorders in a 28-year-old Egyptian woman. Biol Med Case Rep. 2018;2(2):9-11.

* Dexa scan revealed severe osteoporosis (T-score: 4.5 )

* Upper endoscopy: Multiple mucosal tears.

* Nerve conduction of both lower limbs was normal.

* Audiogram: Conductive as well as sensory neural deafness.

* Fundus examination: Bilateral optic cooing vitreous opacities with right papilledema.

Later she started to complain of daily night recurrent temporal severe headache for 10 to 15 minutes in addition to the continuous recurrent night fever which didn't relate to any possible infection proved by the according investigations. A provisional diagnosis at that time of a combination between Osteogenesis imperfect and Ehlers Danlos syndrome (hypermobile type) was out and genetic testing with muscle biopsy were asked for [6]. The patient refused the muscle biopsy and for financial issues only the genetic testing of COL2A1 gene was performed awhile COL1A1/COL1A2 were cancelled socially after review of literature that the diagnosis of osteogenesis imperfect and Ehlers Danlos syndrome was sufficiently made by history and clinical examination. COL2A1 was donea whole exam a sequencing (centroXome Gold) was Performed. Genetic results showed heterogeneous variant and the genetic diagnosis was COL2A1 related disorder as shown in the following Figure 4.

MRI brain showed widening in the subarachnoid solace around the optic nerves and brain atrophy. MRI for CSF flowmetry (imaging study of the CSF few technique used is time-resolved 2D phase contrast MRI with velocity encoding) was normal [7].

* The previous provisional diagnosis was out on the clear history and the clinic examination.

* The recurrent fever was explained in the presence of the hypermobile type of Ehlers Danlos Syndrome as the TNF receptor associated periodic fever syndrome (TRAP) syndrome confirmed by complete resolution of fever for the first time after months by the use of the TNF blocker Adalimumab [8].

* The short stature depressed nasal bridge enlarged skull waddling gait (due to lower limbs dysplasia proved radio logically) a vitreous

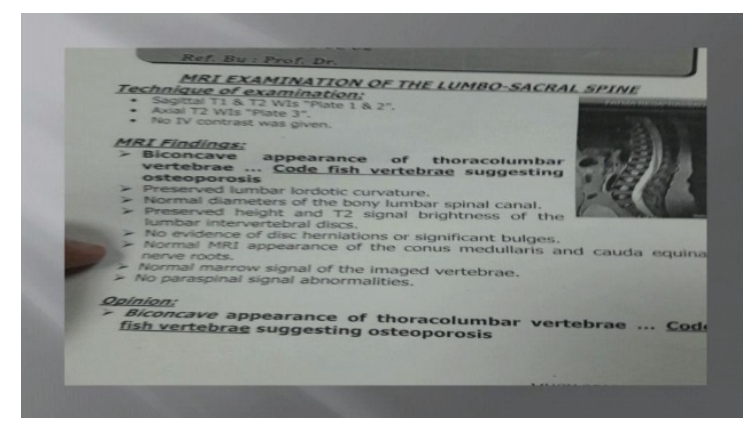

Figure 3. Dexa scan revealed severe osteoporosis.

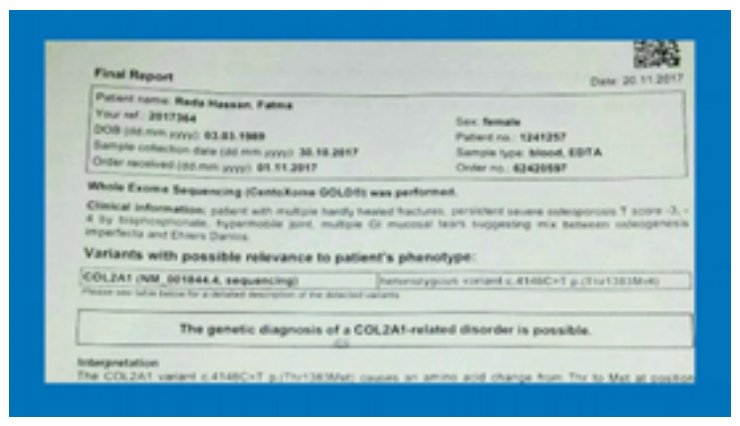

Figure 4. Heterogenous variant and the genetic diagnosis. opacities optic nerve papilledema conductive as well as sensory neural deafness all of the above confirmed by the mutated COL2A1 gene confirm also the presence of Stickler syndrome also in addition to ontogenesis imperfect a Ehlers Danlosa and TRAP syndromes [9].

\section{Discussion}

The constitution of the normal connective tissue varies from one tissue to other both in the specific elements and in the proportion of each element [10]. In addition mutations in genes that affect protein glycolisation are also implicated in the differential diagnosis of connective tissue disorders (1a2). This report describes a woman with multiple hereditary connective tissue disorders didn't present at one patient before. We diagnosed this patient as a combination between osteogenesis imperfect Ehlers Danlos syndrome (hypermobile type) a TRAP syndrome and Stickler syndrome. Several cases had been reported osteogenesis imperfect and also several cases as Ehlers Danlos syndrome with its various types including the hyper mobile type and the vascular type few cases were reported as a combination between osteogenesis imperfect and Ehlers Danlos syndromes [11]. Collagen type 2 aloha 1 diseases are a group of hereditary connective tissue disorders due to mutations in COL2A1 gene. They comprise a group of disorders of dysplasia's and rare syndromes including the Stickler syndrome (4a7). In this patient the diagnosis of osteogenesis imperfect is made by history of multiple recurrent fractures following minor trauma are current low bone mineral density on DEXA scanning in spite of continuous treatment by several lines like calcitonin abisohosohonatesa and finally denosumab with minor improvement and blue sclera on examination (5a6).

Several clinical manifestations at this woman suggest Ehlers Danlos syndrome. In context of the hypermobile joints and elastic skin;the hyper mobile type is more prominent about the recurrent melena and the multiple mucosal tears detected by endoscopy refers to an overload with vascular type Asia a $\mathrm{CT}$ angiography for brain aches and abdomen were done searching for oossible aneurysms or vascular malformations but nothing like this was found [12]. Genetic testing prove mutations in $C O L 2 A 1$ gene favoring $C O L 2 A 1$ related disorders a which describes well what is found in this patient in terms of skeletal abnormalities short stature enlarged skull depressed nasal bridge a optic nerve abnormalities a vitreous ooacitiesaand conductive as well as sensory neural deafness. These manifestations as well as Genetic testing results fatted well with the Stickler syndrome (4a7). The four months history of the recurrent night fever after excluding all the causes of infections as well as other immunological causes suggested high possibility of TNF receptor associated periodic fever (TRAP syndrome)a which is reported to be associated to the hypermobile type of Ehlers Danlos syndrome (8a9a10) socially after complete resolution of this fever after receiving the TNF blocker adalimumab after four months of fever. The Dizzy Spills is explained by autonomic neuropathy commonly occurred with Ehlers Danlos syndrome. (9a10). Throughout the course of treatment the care manager would provide support to the patient in implementing actions based on the GP recommendations or in taking steps to make the lifestyle changes needed to improve health or lower health risk. Unfortunately an in this case like many others we don't have this system of care manager asocial workers actually a mother is doing well the role of care manager [13].

\section{Management}

* During the episodes of melena the patient is made nothing per mouth (NPO) and received Antiemetic's blood transfusion and total parenteral nutrition till her general conditions returned normal again.

* Vitamin D replacement asymptomatic treatment of fever and headache consideration of TRAP syndrome then she took adalimumab subcutaneous injection every 15 days with excellent response regarding the control of fever. 
* $\quad$ For the attacks of recurrent night temporal headache a search for increased intracranial tension was tried about the patient refused to do a CSF manometer and an ethical committee was held for convincing her to complete the investigations in this issue but she is continuing to refuse and also a search for CSF obstruction was done by MRI flowmetry for the CSF it showed no obstruction to the CSF pathway. The papilledema detected as well as the optic nerve cooing is explained by both the Stickler syndrome and Ehlers Danlos syndrome in addition to the possibility of increased intracranial tension.

\section{Conclusion}

Multiple hereditary rare connective tissue disorders are present in a 28-year-Egyptian woman. Up to our knowledge and review this is the first case worldwide comprise the existence of four rare hereditary connective tissue syndromes at the same time in one patient.

\section{References}

1. Pepmueller H. Undifferentiated connective tissue disease, mixed connective tissue disease, and overlap syndromes in rheumatology. Mo Med. 2016;113:136-40.

2. Malfait FR, Symoens S, Coucke P, et al. Total absence of the $\alpha 2$ (I) chain of collagen type I causes a rare form of Ehlers-Danlos syndrome with hypermobility and propensity to cardiac valvular problems. J Med Genet. 2006;43(7):36.

3. Germain DP. Clinical and genetic features of vascular EhlersDanlos syndrome. Ann Vasc Surg. 2002; 16(3):391-7.

4. Liberfarb RM, Levy HP, Rose PS, et al. The Stickler syndrome: Genotype/phenotype correlation in 10 families with Stickler syndrome resulting from seven mutations in the type II collagen gene locus COL2A1. Genet Med. 2003;5(1):21.

5. Barnes AM, Chang W, Morello R, et al. Deficiency of cartilage-associated protein in recessive lethal osteogenesis imperfecta. New Engl J Med. 2006;355(26):2757-64.
6. Lapunzina P, Aglan M, Temtamy S, et al. Identification of a frameshift mutation in Osterix in a patient with recessive osteogenesis imperfecta. Am J Hum Genet. 2010;87(1):110-4.

7. Donoso LA, Edwards AO, Frost AT, et al. Clinical variability of Stickler syndrome. Surv Ophthalmol. 2003;48(2):191-203.

8. Yao Q, Zhou L, Tomecki KJ. Coexistent tumor necrosis factor receptor-associated periodic fever syndrome and EhlersDanlos syndrome. Rheumatol Int. 2012;32(7):2223-5.

9. Kriegel MA. Tumor necrosis factor receptor associated periodic syndrome characterized by a mutation affecting the cleavage site of the receptor. Implications for pathogenesis. Arthritis Rheum. 2003;48(8): 2386-2388.

10. Kriegel MA, Hüffmeier U, Scherb E, et al. Tumor necrosis factor receptor-associated periodic syndrome characterized by a mutation affecting the cleavage site of the receptor: Implications for pathogenesis. Arthritis Rheumatol. 2003;48(8):2386-8.

11. Malfait F, Symoens S, Goemans N, et al. Helical mutations in type I collagen that affect the processing of the aminopropeptide result in an Osteogenesis Imperfecta/EhlersDanlos Syndrome overlap syndrome. Orphanet J Rare Dis. 2013;8(1):78.

12. Ciccone MM, Aquilino A, Cortese F, et al. Feasibility and effectiveness of a disease and care management model in the primary health care system for patients with heart failure and diabetes (Project Leonardo). Vascular health and risk management. 2010;6:297.

13. Murphy-Ryan M, Psychogios A, Lindor NM. Hereditary disorders of connective tissue: A guide to the emerging differential diagnosis. Genet Med. 2010;12(6):344.

\section{*Correspondence to:}

\section{El-Saadany $\mathrm{H}$}

Department of Internal Medicine and Rheumatology unit Air Force Specialized Hospital

New Cairo

Egypt

Tel: +20 19448

E-mail: hanyelsadany@yahoo.com 